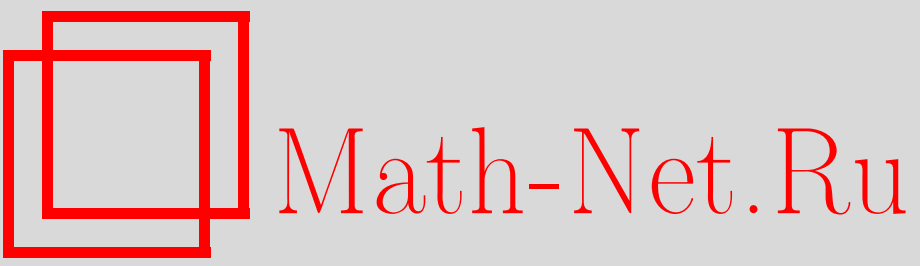

В. И. Денисов, Исследование эффективного пространства-времени нелинейной электродинамики вакуума в поле магнитного диполя, ТМФ, 2002, том 132, номер 2, 211-221

DOI: https://doi.org/10.4213/tmf357

Использование Общероссийского математического портала Math-Net.Ru подразумевает, что вы прочитали и согласны с пользовательским соглашением

http://www . mathnet.ru/rus/agreement

Параметры загрузки:

IP : 3.89 .185 .249

26 апреля 2023 г., 04:03:59 
ТЕОРЕТИЧЕСКАЯ

И МАТЕМАТИЧЕСКАЯ

ФИЗИКА

Том 132, № 2

август, 2002

(C) 2002 г.

В. И. Денисов*

\section{ИССЛЕДОВАНИЕ ЭФФЕКТИВНОГО ПРОСТРАНСТВА-ВРЕМЕНИ НЕЛИНЕЙНОЙ ЭЛЕКТРОДИНАМИКИ ВАКУУМА В ПОЛЕ МАГНИТНОГО ДИПОЛЯ}

В параметризованной постмаксвелловской электродинамике вакуума найден метрический тензор эффективного псевдориманова пространства-времени для электромагнитной волны, распространяюшейся в магнитном дипольном и гравитационном полях нейтронной звезды. На основе анализа изотропных геодезических этого пространства вычислены углы нелинейно-электродинамического и гравитационного искривлений лучей для электромагнитных волн, распространяющихся в плоскости магнитного экватора звезды.

Показано, что для всех нелинейных теорий, у которых постмаксвелловские параметры не совпадают, скорость распространения электромагнитных сигналов во внешних полях и лучи, по которым они распространяются, зависят от поляризации электромагнитных волн. Для двух главных поляризационных состояний электромагнитных сигналов вычислена разность времен распространения этих сигналов от источника до детектора.

Ключевые слова: нелинейная электродинамика вакуума, эффективное пространство-время, нейтронная звезда, искривление лучей.

\section{1. ВВЕДЕНИЕ}

Как известно [1], [2], любому физическому полю соответствует некоторая геометрия, называемая естественной, именно такая, что в отсутствие взаимодействия с другими полями фронт свободной волны этого физического поля движется по геодезическим естественного пространства-времени. Вопрос о выборе естественной геометрии - это вопрос о том, посредством какого эффективного метрического тензора свертываются старшие производные в плотности лагранжиана. Вполне возможна отмечавшаяся еше Лобачевским [3] ситуация, когда различные физические явления будут описываться в терминах различных естественных геометрий.

Поэтому в сформулированных в некотором псевдоримановом пространстве-времени нелинейных моделях, содержаших нелинейность при старших производных, распрос-

* Московский государственный университет, Москва, Россия. E-mail: denisov@srd.sinp.msu.ru 
транение фронта волны физического поля будет происходить по геодезическим эффективного пространства-времени, метрический тензор которого зависит от метрического тензора исходного псевдориманова пространства-времени и полевых переменных этого физического поля.

Все это в полной мере относится и к нелинейной теории электромагнитного поля. В теории поля рассматривается несколько различных моделей нелинейной электродинамики вакуума. В случае слабых полей их лагранжиан в псевдоевклидовом пространстве-времени может быть записан [4] в параметризованном постмаксвелловском виде, аналогичном постньютоновскому разложению [5], применяющемуся в теории гравитации:

$$
\begin{aligned}
L= & \frac{1}{8 \pi}\left\{\left(\mathbf{E}^{2}-\mathbf{B}^{2}\right)+\xi\left[\eta_{1}\left(\mathbf{E}^{2}-\mathbf{B}^{2}\right)^{2}+4 \eta_{2}(\mathbf{B E})^{2}\right]\right\}+ \\
& +O\left(\eta \xi^{2} \mathbf{B}^{6}\right)-\rho \varphi+\frac{1}{c}(\mathbf{j} \mathbf{A}) .
\end{aligned}
$$

Здесь $\xi=1 / B_{q}^{2}, \quad B_{q}=m^{2} c^{3} / e \hbar=4.41 \cdot 10^{13} \Gamma c$ - характерное квантовоэлектродинамическое значение, а величина безразмерных постмаксвелловских параметров $\eta_{1}$ и $\eta_{2}$ зависит от выбора модели нелинейной электродинамики вакуума.

В частности, в нелинейной электродинамике Гейзенберга-Эйлера [6] параметры $\eta_{1}$ и $\eta_{2}$ имеют вполне конкретные значения $\eta_{1}=\alpha /(45 \pi)=5.1 \cdot 10^{-5}, \eta_{2}=7 \alpha /(180 \pi)=$ $9.0 \cdot 10^{-5}$, в то время как в теории Борна-Инфельда [7] $\eta_{1}=\eta_{2}=a^{2} B_{q}^{2} / 4$, причем для постоянной $a^{2}$ известна лишь оценка снизу: $a^{2}>1.2 \cdot 10^{-32} \Gamma^{-2}$.

Наиболее ярко нелинейные свойства электродинамики вакуума должны проявляться в сильных электромагнитных полях. Такие поля макроскопических размеров в природе сушествуют только в окрестности нейтронных звезд. В частности, астрофизические наблюдения показывают, что у многих пульсаров магнитное поле на поверхности достигает значений $10^{12} \div 10^{13} \Gamma$, а у недавно открытых магнетаров $10^{15} \Gamma$.

По этой причине мы будем рассматривать основные нелинейно-электродинамические эффекты, которые возникают при распространении электромагнитных волн в магнитном поле нейтронных звезд.

Так как нейтронные звезды обладают значительным гравитационным полем, то выражение (1) следует записать в обшековариантном виде:

$$
L=\frac{\sqrt{-g}}{32 \pi}\left\{2 J_{2}+\xi\left[\left(\eta_{1}-2 \eta_{2}\right) J_{2}^{2}+4 \eta_{2} J_{4}\right]\right\}+O\left(\eta \xi^{2} \mathbf{B}^{6}\right)-\frac{\sqrt{-g}}{c} j^{n} A_{n},
$$

где $g$ - определитель метрического тензора исходного псевдориманова пространствавремени, а $J_{2}=F_{i k} F^{k i}$ и $J_{4}=F_{i k} F^{k m} F_{m l} F^{l i}$ - независимые инварианты тензора электромагнитного поля $F_{i k}$.

Система уравнений электромагнитного поля нелинейной электродинамики вакуума с плотностью лагранжиана (2) имеет вид

$$
\begin{gathered}
\frac{1}{\sqrt{-g}} \frac{\partial}{\partial x^{n}}\left\{\sqrt{-g} Q^{m n}\right\}=-\frac{4 \pi}{c} j^{m}, \\
\frac{\partial F_{m n}}{\partial x^{k}}+\frac{\partial F_{n k}}{\partial x^{m}}+\frac{\partial F_{k m}}{\partial x^{n}}=0,
\end{gathered}
$$


где для сокрашения записи введено обозначение

$$
Q^{m n}=\left[1+\xi\left(\eta_{1}-2 \eta_{2}\right) J_{2}\right] F^{m n}+4 \xi \eta_{2} F^{m l} F_{l k} F^{k n}
$$

Найдем метрический тензор эффективного пространства-времени нелинейной электродинамики вакуума с плотностью функции Лагранжа (2) и проанализируем основные эффекты, которые возникают при прохождении слабой электромагнитной волны через магнитное дипольное и гравитационное поля нейтронной звезды. В качестве метрического тензора исходного псевдориманова пространства-времени будем использовать решение Шварцшильда

$$
g_{00}=1-\frac{r_{g}}{r}, \quad g_{r r}=-\frac{r}{r-r_{g}}, \quad g_{\theta \theta}=-r^{2}, \quad g_{\varphi \varphi}=-r^{2} \sin ^{2} \theta
$$

где $r_{g}$ - гравитационньй радиус нейтронной звезды.

В рассматриваемой нами задаче имеются два малых параметра: $r_{g} / r$ и $\eta_{1,2} \xi \mathbf{B}^{2}$. Проведем вычисление основных нелинейно-электродинамических и гравитационных эффектов с квадратичной точностью по параметру $r_{g} / r$ и линейной по параметру $\eta_{1,2} \xi \mathbf{B}^{2}$.

\section{2. УРАВНЕНИЕ ГАМИЛЬТОНА-ЯКОБИ}

В линейном приближении решение уравнений (3), описываюшее дипольное магнитное поле нейтронной звезды, имеет вид

$$
F_{31}^{(0)}=-\frac{|\mathbf{m}|}{r^{2}} \sin ^{2} \theta, \quad F_{32}^{(0)}=\frac{2|\mathbf{m}|}{r} \sin \theta \cos \theta
$$

где $\mathbf{m}$ - магнитньй дипольный момент.

Найдем теперь уравнение Гамильтона-Якоби, которому удовлетворяют лучи слабой высокочастотной электромагнитной волны, распространяющейся в гравитационном (4) и магнитном (5) полях нейтронной звезды.

Тензор электромагнитного поля, входящий в уравнения (3), в этом случае следует представить в виде суммы дипольного магнитного поля $F_{i k}^{(0)}$ звезды $(5)$ и поля слабой электромагнитной волны $f_{i k}: F_{i k}=F_{i k}^{(0)}+f_{i k}$.

В линейном приближении по слабой электромагнитной волне $f_{i k}$ уравнения (3) принимают вид

$$
\begin{gathered}
\frac{1}{\sqrt{-g}} \frac{\partial}{\partial x^{n}}\left\{\sqrt{-g} Q_{(1)}^{m n}\right\}=0 \\
\frac{\partial f_{m n}}{\partial x^{k}}+\frac{\partial f_{n k}}{\partial x^{m}}+\frac{\partial f_{k m}}{\partial x^{n}}=0
\end{gathered}
$$

где

$$
\begin{aligned}
Q_{(1)}^{m n}= & {\left[1+\xi\left(\eta_{1}-2 \eta_{2}\right) J_{2}^{(0)}\right] f^{m n}+4 \xi \eta_{2}\left[f^{m l} F_{l k}^{(0)} F_{(0)}^{k n}+\right.} \\
& \left.+F_{(0)}^{m l} f_{l k} F_{(0)}^{k n}+F_{(0)}^{m l} F_{l k}^{(0)} f^{k n}\right]+\left[2 \xi\left(\eta_{1}-2 \eta_{2}\right) f_{i k} F_{(0)}^{k i}\right] F_{(0)}^{m n}
\end{aligned}
$$


Так как нас интересует прежде всего воздействие гравитационного и магнитного полей звезды на лучи слабой электромагнитной волны, то, используя систему уравнений (6), найдем уравнение, которому должен удовлетворять в рассматриваемом случае эйконал. Для этого представим тензор $f_{n m}$ слабой электромагнитной волны в виде

$$
f_{n m}=A_{n m}(\mathbf{r}, t) e^{i S(\mathbf{r}, t)}
$$

где, как обычно, амплитуда $A_{n m}(\mathbf{r}, t)$ является медленно изменяющейся функцией координат и времени, а эйконал $S(\mathbf{r}, t)$ - быстро изменяющейся функцией.

В результате производные от $S$ оказываются чрезвычайно большими величинами, так что выполняется условие

$$
\left|A_{n m}(\mathbf{r}, t) \frac{\partial S}{\partial x^{l}}\right| \gg\left|\frac{\partial A_{n m}(\mathbf{r}, t)}{\partial x^{l}}\right| .
$$

Подставим выражение (7) в уравнения (6). Учтем, что для точек, находяшихся вне звезды, по порядку величины выполняются соотношения

$$
\frac{\partial F_{n m}^{(0)}}{\partial r} \sim \frac{F_{n m}^{(0)}}{R}, \quad\left|\frac{\partial g_{n m}^{(0)}}{\partial r}\right| \ll\left|\frac{g_{n m}^{(0)}}{R}\right|, \quad \frac{\partial S}{\partial r} \sim \frac{S}{\lambda},
$$

где $R$ - радиус звезды, $\lambda$ - длина волны.

Так как для высокочастотного электромагнитного излучения $R / \lambda \gg 1$, то при дифференцировании $Q_{(1)}^{n m}$ в уравнениях (6) следует оставить только производные от эйконала $S$. В результате линейно-независимые уравнения системы (6) примут вид однородной системы линейных алгебраических уравнений:

$$
\begin{aligned}
{[1+} & \left.\xi\left(\eta_{1}-2 \eta_{2}\right) J_{2}^{(0)}\right] f^{\mu n} \frac{\partial S}{\partial x^{n}}+ \\
& +4 \xi \eta_{2}\left[f^{\mu l} F_{l k}^{(0)} F_{(0)}^{k n}+F^{\mu l} f_{l k} F_{(0)}^{k n}+F_{(0)}^{\mu l} F_{l k}^{(0)} f^{k n}\right] \frac{\partial S}{\partial x^{n}}+ \\
& +\left[2 \xi\left(\eta_{1}-2 \eta_{2}\right) f_{i k} F_{(0)}^{k i}\right] F_{(0)}^{\mu n} \frac{\partial S}{\partial x^{n}}=0, \\
f_{\alpha \beta} & \frac{\partial S}{\partial x^{0}}+f_{\beta 0} \frac{\partial S}{\partial x^{\alpha}}+f_{0 \alpha} \frac{\partial S}{\partial x^{\beta}}=0 .
\end{aligned}
$$

Домножая первое уравнение системы (8) на $\partial S / \partial x^{0}$ и исключая из него компоненты $f_{\alpha \beta}$ с помошью второго уравнения, приходим к системе уравнений вида

$$
\Pi^{\mu \beta} f_{0 \beta}=0
$$

Уравнение эйконала, как известно, является следствием условия

$$
\operatorname{det}\left\|\Pi^{\mu \beta}\right\|=0 \text {. }
$$

Учитывая, что в нашей задаче отличными от нуля компонентами тензора $F_{i k}^{(0)}$ являются только компоненты (5), и используя алгоритм, развитый в работах [8]-[10], после 
сокращения на несущественный множитель получаем следуюшее уравнение Гамильтона-Якоби:

$$
\begin{aligned}
\left\{\frac{r}{r-r_{g}}\right. & \left(\frac{\partial S}{\partial x^{0}}\right)^{2}-\left(1-\frac{r_{g}}{r}\right)\left(\frac{\partial S}{\partial r}\right)^{2}-\frac{1}{r^{2}}\left(\frac{\partial S}{\partial \theta}\right)^{2}- \\
& -\frac{1}{r^{2} \sin ^{2} \theta}\left(\frac{\partial S}{\partial \varphi}\right)^{2}+\frac{4 \eta_{1} \xi \mathbf{m}^{2}}{r^{6}}\left[\left(\sin \theta \frac{\partial S}{\partial r}-\frac{2 \cos \theta}{r} \frac{\partial S}{\partial \theta}\right)^{2}+\right. \\
& \left.\left.+\frac{1+3 \cos ^{2} \theta}{r^{2} \sin ^{2} \theta}\left(\frac{\partial S}{\partial \varphi}\right)^{2}\right]+O\left(\eta \xi^{2} B^{4}\right)\right\} \times \\
& \times\left\{\frac{r}{r-r_{g}}\left(\frac{\partial S}{\partial x^{0}}\right)^{2}-\left(1-\frac{r_{g}}{r}\right)\left(\frac{\partial S}{\partial r}\right)^{2}-\frac{1}{r^{2}}\left(\frac{\partial S}{\partial \theta}\right)^{2}-\right. \\
& -\frac{1}{r^{2} \sin ^{2} \theta}\left(\frac{\partial S}{\partial \varphi}\right)^{2}+\frac{4 \eta_{2} \xi \mathbf{m}^{2}}{r^{6}}\left[\left(\sin \theta \frac{\partial S}{\partial r}-\frac{2 \cos \theta}{r} \frac{\partial S}{\partial \theta}\right)^{2}+\right. \\
& \left.\left.+\frac{1+3 \cos ^{2} \theta}{r^{2} \sin ^{2} \theta}\left(\frac{\partial S}{\partial \varphi}\right)^{2}\right]+O\left(\eta \xi^{2} B^{4}\right)\right\}=0
\end{aligned}
$$

Таким образом, в зависимости от поляризации слабой электромагнитной волны ее эйконал будет удовлетворять одному из двух уравнений Гамильтона-Якоби:

$$
\begin{aligned}
\frac{r}{r-r_{g}} & \left(\frac{\partial S}{\partial x^{0}}\right)^{2}-\left(1-\frac{r_{g}}{r}\right)\left(\frac{\partial S}{\partial r}\right)^{2}-\frac{1}{r^{2}}\left(\frac{\partial S}{\partial \theta}\right)^{2}- \\
& -\frac{1}{r^{2} \sin ^{2} \theta}\left(\frac{\partial S}{\partial \varphi}\right)^{2}+\frac{4 \eta_{1,2} \xi \mathbf{m}^{2}}{r^{6}}\left[\left(\sin \theta \frac{\partial S}{\partial r}-\frac{2 \cos \theta}{r} \frac{\partial S}{\partial \theta}\right)^{2}+\right. \\
& \left.+\frac{1+3 \cos ^{2} \theta}{r^{2} \sin ^{2} \theta}\left(\frac{\partial S}{\partial \varphi}\right)^{2}\right]=O\left(\eta \xi^{2} B^{4}\right),
\end{aligned}
$$

где $\eta_{1,2}=\eta_{2}$ для электромагнитной волны, поляризованной в плоскости, содержашей векторы $\mathbf{B}$ и $\mathbf{k}$, и $\eta_{1,2}=\eta_{1}$ для электромагнитной волны, поляризованной перпендикулярно к этой плоскости.

\section{3. ЭФФЕКТИВНАЯ МЕТРИКА ЭЛЕКТРОДИНАМИКИ В ПОЛЕ МАГНИТНОГО ДИПОЛЯ}

Сравнивая уравнение (9) с уравнением Гамильтона-Якоби в общем виде

$$
g^{m n} \frac{\partial S}{\partial x^{m}} \frac{\partial S}{\partial x^{n}}=0
$$

найдем компоненты метрического тензора эффективного пространства-времени для электромагнитных волн, распространяюшихся в поле нейтронной звезды:

$$
\begin{gathered}
g^{00}=\frac{r}{r-r_{g}}, \quad g^{r r}=-1+\frac{r_{g}}{r}+\frac{4 \eta_{1,2} \xi \mathbf{m}^{2}}{r^{6}} \sin ^{2} \theta \\
g^{\theta \theta}=-\frac{1}{r^{2}}\left(1-\frac{16 \eta_{1,2} \xi \mathbf{m}^{2}}{r^{6}} \cos ^{2} \theta\right), \quad g^{r \theta}=-\frac{8 \eta_{1,2} \xi \mathbf{m}^{2}}{r^{7}} \sin \theta \cos \theta \\
g^{\varphi \varphi}=-\frac{1}{r^{2} \sin ^{2} \theta}\left[1-\frac{4 \eta_{1,2} \xi \mathbf{m}^{2}}{r^{6}}\left(1+3 \cos ^{2} \theta\right)\right] .
\end{gathered}
$$


Используя эти выражения, несложно вычислить компоненты тензора Риччи для пространства с метрическим тензором (10):

$$
\begin{array}{ll}
R_{r r}=-\frac{24 \eta_{1,2} \xi \mathbf{m}^{2}\left(5+12 \cos ^{2} \theta\right)}{r^{8}}, & R_{r \theta}=-\frac{48 \eta_{1,2} \xi \mathbf{m}^{2} \sin \theta \cos \theta}{r^{7}}, \\
R_{\theta \theta}=-\frac{12 \eta_{1,2} \xi \mathbf{m}^{2}\left(3+7 \cos ^{2} \theta\right)}{r^{6}}, & R_{\varphi \varphi}=-\frac{60 \eta_{1,2} \xi \mathbf{m}^{2}\left(1+\cos ^{2} \theta\right) \sin ^{2} \theta}{r^{6}} .
\end{array}
$$

Отметим, что скалярная кривизна этого пространства также отлична от нуля:

$$
R=R_{i k} g^{i k}=\frac{216 \eta_{1,2} \xi \mathbf{m}^{2}\left(1+2 \cos ^{2} \theta\right)}{r^{8}}
$$

Следует, однако, подчеркнуть, что эффективное пространство-время при $\eta_{1} \neq \eta_{2}$ не является универсальным даже для электродинамики, так как его воздействие на распространение электромагнитных волн зависит от их состояния поляризации.

В результате во внешних электромагнитных полях возникает эффект двулучепреломления: скорость распространения электромагнитных волн становится зависяшей от их поляризации. И только в тех нелинейных моделях электродинамики вакуума, где $\eta_{1}=\eta_{2}$, метрический тензор (10) в рассматриваемом приближении становится одинаковым для всех электромагнитных волн независимо от их поляризации. Примером такой теории является теория Борна-Инфельда.

\section{4. УРАВНЕНИЕ СЕМЕЙСТВА ЛУЧЕЙ}

Найдем решение уравнений (9) для лучей, лежаших в плоскости магнитного экватора нейтронной звезды. Поместим начало координат в центр нейтронной звезды и сориентируем ось $z$ вдоль вектора дипольного магнитного момента $\mathbf{m}$. В плоскости магнитного экватора $\theta=\pi / 2$ введем полярные координаты $r$ и $\varphi$.

Тогда уравнение Гамильтона-Якоби для лучей, лежащих в плоскости $\theta=\pi / 2$, принимает вид

$$
\begin{gathered}
\left(1+\frac{r_{g}}{r}+\frac{r_{g}^{2}}{r^{2}}\right)\left(\frac{\partial S}{\partial x^{0}}\right)^{2}-\left(1-\frac{r_{g}}{r}\right)\left(\frac{\partial S}{\partial r}\right)^{2}-\frac{1}{r^{2}}\left(\frac{\partial S}{\partial \varphi}\right)^{2}+ \\
+\frac{4 \eta_{1,2} \xi \mathbf{m}^{2}}{r^{6}}\left[\left(\frac{\partial S}{\partial r}\right)^{2}+\frac{1}{r^{2}}\left(\frac{\partial S}{\partial \varphi}\right)^{2}\right]=0 .
\end{gathered}
$$

Решая это уравнение методом разделения переменных, получим

$$
S=-\mathcal{E}_{0} t+\alpha \varphi \pm \int^{r} d r \sqrt{f(r)}
$$

где знак плюс соответствует распространению электромагнитной волны от нейтронной звезды, а знак минус - к звезде, $\mathcal{E}_{0}$ и $\alpha$ - произвольные постоянные и введено обозначение

$$
f(r)=\frac{\mathcal{E}_{0}^{2}}{c^{2}}\left(1+\frac{2 r_{g}}{r}+\frac{3 r_{g}^{2}}{r^{2}}+\frac{4 \xi \eta_{1,2} \mathbf{m}^{2}}{r^{6}}\right)-\frac{\alpha^{2}}{r^{2}}\left(1+\frac{r_{g}}{r}+\frac{r_{g}^{2}}{r^{2}}\right) .
$$


Рассмотрим некоторый луч электромагнитной волны частоты $\omega_{0}$, начинающийся на пространственной бесконечности и имеюший прицельное расстояние $b_{1,2}$. Если этот луч обходит нейтронную звезду в направлении по часовой стрелке, то константы интегрирования $\mathcal{E}_{0}$ и $\alpha$, входящие в выражения $(11)$ и $(12)$, принимают вид

$$
\mathcal{E}_{0}=\omega_{0}, \quad \alpha=-\frac{\omega_{0} b_{1,2}}{c} .
$$

Уравнение луча в аналитическом виде можно получить, если продифференцировать выражение (11) по $\alpha$, приравнять результат некоторой константе $\varphi_{0}$ и учесть соотношения (13):

$$
\varphi=\varphi_{0} \mp \frac{\omega_{0} b_{1,2}}{c} \int^{r} \frac{d r}{r^{2} \sqrt{f(r)}}\left(1+\frac{r_{g}}{r}+\frac{r_{g}^{2}}{r^{2}}\right) .
$$

Однако исследовать его в таком виде очень сложно. Поэтому продифференцируем равенство (14) по $r$. Переходя к вспомогательной переменной $u=1 / r$, с принятой точностью будем иметь

$$
b_{1,2}^{2}\left(\frac{d u}{d \varphi}\right)^{2}=1+4 \xi \eta_{1,2} \mathbf{m}^{2} u^{6}-b_{1,2}^{2} u^{2}\left(1-r_{g} u\right) .
$$

Из этого выражения несложно найти перицентр луча - минимальное расстояние от нейтронной звезды до луча. Действительно, записывая условие $d u / d \varphi=0$ и решая полученное уравнение методом последовательных приближений, получим

$$
r_{\min }=b_{1,2}\left(1-\frac{r_{g}}{2 b_{1,2}}-\frac{2 \eta_{1,2} \xi \mathbf{m}^{2}}{b_{1,2}^{6}}-\frac{3 r_{g}^{2}}{8 b_{1,2}^{6}}\right) .
$$

Решение уравнения (15) будем искать методом последовательных приближений. В нулевом приближении по малым параметрам $r_{g} u$ и $\xi \mathbf{m}^{2} u^{6}$ это уравнение принимает вид

$$
b_{1,2}^{2}\left(\frac{d u}{d \varphi}\right)^{2}=1-b_{1,2}^{2} u^{2}
$$

Его решением является прямая $u=1 / r=\sin \left(\varphi+\varphi_{0}\right) / b_{1,2}$, проходяшая на расстоянии $b_{1,2}$ от начала координат под углом $-\varphi_{0}$ к оси $x$.

Для получения уравнения луча $u=u(\varphi)$ с большей точностью представим функцию $u$ в виде

$$
u=\frac{1}{r}=\frac{1}{b_{1,2}}[V+W \sin \Psi(\varphi)] .
$$

Подставляя это выражение в уравнение (15) и приравнивая члены, имеющие одинаковый порядок малости, получаем

$$
\begin{gathered}
V_{1,2}=\frac{r_{g}}{2 b_{1,2}}, \quad W_{1,2}=1+\frac{2 \eta_{1,2} \xi \mathbf{m}^{2}}{b_{1,2}^{6}}+\frac{5 r_{g}^{2}}{8 b_{1,2}^{2}}, \\
\Psi(\varphi)_{1,2}=\varphi+\psi_{1,2}+\frac{r_{g}}{2 b_{1,2}} \cos \left(\varphi+\psi_{1,2}\right)- \\
-\frac{r_{g}^{2}}{32 b_{1,2}^{2}}\left[30\left(\varphi+\psi_{1,2}\right)+\sin 2\left(\varphi+\psi_{1,2}\right)\right]- \\
-\frac{\eta_{1,2} \xi \mathbf{m}^{2}}{16 b_{1,2}^{6}}\left[60\left(\varphi+\psi_{1,2}\right)+\sin 4\left(\varphi+\psi_{1,2}\right)-16 \sin 2\left(\varphi+\psi_{1,2}\right)\right],
\end{gathered}
$$


где $\psi_{1,2}-$ постоянная интегрирования.

Для нахождения этой постоянной предположим, что источник гамма-излучения находится в точке $r=1 / u=R_{0}, \varphi=\pi$. Тогда с требуемой точностью приходим к выражению

$$
\begin{aligned}
\psi_{1,2}= & -q_{1,2}+\frac{r_{g}}{2 b_{1,2}} \cos q_{1,2}+\frac{3 r_{g}^{2}}{32 b_{1,2}^{2}}\left[10\left(\pi-q_{1,2}\right)+\sin 2 q_{1,2}\right]+ \\
& +\frac{\eta_{1,2} \xi \mathbf{m}^{2}}{16 b_{1,2}^{6}}\left[60\left(\pi-q_{1,2}\right)-\sin 4 q_{1,2}+16 \sin 2 q_{1,2}\right]
\end{aligned}
$$

где

$$
q_{1,2}=\arcsin \left[\frac{b_{1,2}}{R_{0}}\left(1-\frac{2 \eta_{1,2} \xi \mathbf{m}^{2}}{b_{1,2}^{6}}-\frac{5 r_{g}^{2}}{8 b_{1,2}^{2}}\right)-\frac{r_{g}}{2 b_{1,2}}\right] .
$$

Следует отметить, что источник, находящийся в точке $r=R_{0}, \varphi=\pi$, будет лежать на луче, имеющем прицельное расстояние $b_{1,2}$, если и только если $R_{0} \geqslant r_{\min }$, где $r_{\min }$ определено соотношением (16).

Из выражений (17)-(19) следует, что в тех моделях нелинейной электродинамики вакуума, где $\eta_{1} \neq \eta_{2}$, электромагнитные волны, поляризованные во взаимно перпендикулярных плоскостях и имеющие одинаковые прицельные расстояния $b_{1}=b_{2}$, распространяются по разным лучам.

Найдем углы $\delta \varphi_{1,2}$ нелинейно-электродинамического и гравитационного искривлений лучей в магнитном дипольном и гравитационном полях нейтронной звезды. Предположим, что детектор высокочастотного электромагнитного излучения расположен на рассматриваемом луче с другой стороны нейтронной звезды по отношению к источнику излучения на расстоянии $R_{1}$ от нее. Угловую координату $\varphi_{1,2}$ этого детектора можно найти из трансцендентного уравнения $V+W \sin \Psi\left(\varphi_{1,2}\right)=b_{1,2} / R_{1}$. Решая его методом последовательных приближений по малым параметрам $r_{g} / b_{1,2}$ и $\eta_{1,2} \xi \mathbf{m}^{2} / b_{1,2}^{6}$, получим

$$
\begin{aligned}
\varphi_{1,2}= & p_{1,2}-\psi_{1,2}-\frac{r_{g}}{2 b_{1,2}} \cos p_{1,2}+\frac{3 r_{g}^{2}}{32 b_{1,2}^{2}}\left(10 p_{1,2}-\sin 2 p_{1,2}\right)+ \\
+ & \frac{\eta_{1,2} \xi \mathbf{m}^{2}}{16 b_{1,2}^{6}}\left(60 p_{1,2}+\sin 4 p_{1,2}-16 \sin 2 p_{1,2}\right),
\end{aligned}
$$

где

$$
p_{1,2}=\arcsin \left[\frac{b_{1,2}}{R_{1}}\left(1-\frac{2 \eta_{1,2} \xi \mathbf{m}^{2}}{b_{1,2}^{6}}-\frac{5 r_{g}^{2}}{8 b_{1,2}^{2}}\right)-\frac{r_{g}}{2 b_{1,2}}\right] .
$$

Рассмотрим две электромагнитные волны, одна из которых поляризована в плоскости магнитного экватора нейтронной звезды, а другая - перпендикулярно этой плоскости, распространяющиеся по лучам, имеющим при $R_{0} \rightarrow \infty$ одинаковые прицельные параметры $b_{1}=b_{2}=b$. Подставляя $R_{1}=\infty$ в выражения $(20),(21)$ и ограничиваясь принятой нами точностью, найдем углы искривления этих лучей:

$$
\delta \varphi_{1,2}=-\frac{2 r_{g}}{b}-\frac{15 \pi r_{g}^{2}}{16 b^{2}}-\frac{15 \pi \eta_{1,2} \xi \mathbf{m}^{2}}{4 b^{6}} .
$$


Первые два слагаемых в этом выражении дают гравитационное искривление луча [11], а последнее - нелинейно-электродинамическое [12]. Знак минус в выражении (22) показывает, что гравитационное и магнитное поля нейтронной звезды в плоскости магнитного экватора действуют на электромагнитные волны как собирающая линза.

Угол $\delta \varphi$ между асимптотами этих двух лучей при $R_{1}=\infty$ будет равен

$$
\delta \varphi=\delta \varphi_{1}-\delta \varphi_{2}=\frac{15 \pi\left(\eta_{2}-\eta_{1}\right) \xi \mathbf{m}^{2}}{4 b^{6}}
$$

Таким образом, в нелинейных моделях электродинамики вакуума, для которых $\eta_{2} \neq \eta_{1}$, угол $\delta \varphi \neq 0$.

\section{5. ЗАКОН РАСПРОСТРАНЕНИЯ ЭЛЕКТРОМАГНИТНОГО СИГНАЛА ПО ЛУЧУ}

Закон движения электромагнитного сигнала $T=T(r)$ по лучу (17) можно найти, если продифференцировать выражение $(11)$ по $\mathcal{E}_{0}$, приравнять результат некоторой константе $t_{0}$ и учесть соотношения (13):

$$
T=t_{0} \pm \frac{\omega_{0}}{c^{2}} \int^{r} \frac{d r}{\sqrt{f(r)}}\left(1+\frac{2 r_{g}}{r}+\frac{3 r_{g}^{2}}{r^{2}}+\frac{4 \xi \eta_{1,2} \mathbf{m}^{2}}{r^{6}}\right)
$$

Дифференцируя последнее равенство по $r$ и учитывая выражения (15) и (18), несложно получить

$$
\begin{aligned}
\frac{d T}{d \Psi}= & -\frac{1}{c b_{1,2} u^{2}}\left(1+\frac{2 \eta_{1,2} \xi \mathbf{m}^{2}}{b_{1,2}^{6}}+\frac{r_{g}^{2}}{2 b_{1,2}^{2}}\right)-\frac{3 r_{g}}{2 c b_{1,2} u}- \\
& -\frac{b_{1,2}}{c}\left[\frac{2 \eta_{1,2} \xi \mathbf{m}^{2}}{b_{1,2}^{6}}\left(1+\sin ^{2} \Psi+2 \sin ^{4} \Psi\right)+\frac{15 r_{g}^{2}}{8 b_{1,2}^{2}}\right] .
\end{aligned}
$$

Интегрируя это уравнение, находим

$$
\begin{aligned}
T= & t_{1,2}-\frac{r_{g}}{c} \ln \left|V \operatorname{tg} \frac{\Psi(\varphi)}{2}+W-\sqrt{W^{2}-V^{2}}\right|+ \\
& +\frac{r_{g}}{c} \ln \left|V \operatorname{tg} \frac{\Psi(\varphi)}{2}+W+\sqrt{W^{2}-V^{2}}\right|+\frac{r}{c}\left(1+\frac{r_{g}^{2}}{8 b_{1,2}^{2}}\right) \cos \Psi(\varphi)- \\
& -\frac{b_{1,2}}{c}\left\{\frac{\eta_{1,2} \xi \mathbf{m}^{2}}{8 b_{1,2}^{6}}[36 \Psi(\varphi)-12 \sin 2 \Psi(\varphi)+\sin 4 \Psi(\varphi)]+\frac{15 r_{g}^{2}}{8 b_{1,2}^{2}} \Psi(\varphi)\right\}
\end{aligned}
$$

где $t_{1}$ и $t_{2}-$ постоянные интегрирования.

Следует отметить, что $T$ - время, измеряемое по часам наблюдателя, находяшегося на значительном удалении от нейтронной звезды $\left(r_{g} / R_{1} \rightarrow 0\right)$. При $r_{g} \rightarrow 0$ и $\mathbf{m}^{2} \rightarrow 0$ выражение (23), как и следовало ожидать, описывает движение электромагнитного сигнала со скоростью $v=c$ по прямой, проходящей на расстоянии $b_{1,2}$ от начала координат. 
Из выражения (23) следует, что в плоскости магнитного экватора при $\eta_{1} \neq \eta_{2}$ два электромагнитных сигнала, поляризованные во взаимно перпендикулярных плоскостях (один в плоскости магнитного экватора, а другой - перпендикулярно этой плоскости) и излученные в один и тот же момент времени из одного и того же источника, прибудут к наблюдателю по различным лучам и в различные моменты времени $T_{1} \neq T_{2}$.

Как показывает детальный анализ, если источник высокочастотного излучения находится вблизи нейтронной звезды, то асимптотически главный член разности $\Delta T=T_{2}-$ $T_{1}$ принимает вид

$$
\Delta T=\frac{9 \pi\left(\eta_{2}-\eta_{1}\right) \xi \mathbf{m}^{2}}{4 b^{5} c}
$$

Величина $\Delta T$ сушественно зависит от разности постмаксвелловских параметров. В частности, в нелинейной электродинамике Борна-Инфельда $\Delta T=0$, в то время как согласно электродинамике Гейзенберга-Эйлера выражение (24) в магнитных полях пульсаров и магнетаров может достигать величины $10^{-6}$ с. Современная электроника в состоянии зарегистрировать сигналы различной поляризации, разделенные такими промежутками времени.

\section{6. ЗАКЛЮЧЕНИЕ}

Таким образом, наличие нелинейных слагаемых при старших производных в лагранжиане электромагнитного поля приводит к появлению эффективного псевдориманова пространства-времени, метрический тензор которого зависит не только от внешнего электромагнитного поля, но и от поляризации электромагнитной волны, распространяющейся в этом поле.

В результате внешнее электромагнитное поле оказывает на электромагнитные волны нелинейно-электродинамическое действие, во многом аналогичное действию гравитационного поля: лучи электромагнитных волн в неоднородном электромагнитном поле искривляются, а скорость распространения электромагнитных сигналов зависит не только от внешнего поля, но и от их поляризации.

Это нелинейно-электродинамическое воздействие происходит на фоне обычного гравитационного воздействия. Поэтому при проведении измерений различных гравитационных эффектов, происходящих в окрестностях пульсаров и магнетаров, необходимо проводить также поиск и измерение нелинейно-электродинамических эффектов.

Полученные при таких измерениях результаты позволят выяснить экспериментальньй статус различных моделей нелинейной электродинамики вакуума и исследовать естественную геометрию для электромагнитного поля в нелинейной электродинамике вакуума.

Благодарности. Работа выполнена при поддержке РФФИ, грант № 02-02-16598. 


\section{Список литературы}

[1] A. А. Логунов. Лекции по теории относительности и гравитации. М.: Наука, 1987.

[2] В. И. Денисов, М. И. Денисов, Н. В. Кравцов, В. Б. Пинчук. ТМФ. 1998. Т. 117. № 3. C. 498.

[3] Н. И. Лобачевский. Полное собрание сочинений. Т. 2. М.: Гостехиздат, 1949.

[4] В. И. Денисов, И. П. Денисова. Докл. РАН. 2001. Т. 378. № 4. С. 463.

[5] K. Уилл. Теория и эксперимент в гравитационной физике. М.: Энергоатомиздат, 1985.

[6] W. Heisenberg, H. Euler. Z. Phys. 1936. V. 26. P. 714.

[7] M. Born, L. Infeld. Proc. Roy. Soc. London. Ser. A. 1934. V. 144. P. 425.

[8] V. I. Denisov, M. I. Denisov. Phys. Rev. D. 1999. V. 60. № 4. P. 047301.

[9] V. I. Denisov. Phys. Rev. D. 2000. V. 61. № 3. P. 036004.

[10] В. И. Денисов, И. П. Денисова. Оптика и спектроскопия. 2001. Т. 90. № 2. С. 329.

[11] R. Epstein, I. I. Shapiro. Phys. Rev. D. 1980. V. 22. P. 2947.

[12] В. И. Денисов, И. П. Денисова, С. И. Свертилов. Докл. РАН. 2001. Т. 380. С. 754.

Поступила в редакцию 15.III.2002 г. 